\title{
IDENTIFICAÇÃO E AVALIAÇÃO DO PERFIL DE RESISTÊNCIA DE BACTÉRIAS ISOLADAS DA UNIDADE DE TERAPIA INTENSIVA DE UM HOSPITAL DA REGIÁO NOROESTE DO RIO GRANDE DO SUL
}

\author{
IDENTIFICATION AND EVALUATION OF THE RESISTANCE PROFILE OF \\ ISOLATED BACTERIA OF THE INTENSIVE CARE UNIT OF A HOSPITAL \\ IN THE NORTHWEST REGION OF RIO GRANDE DO SUL
}

Graciele Vanessa Wisniewski ${ }^{1}$, Tanise Maria Fiorin ${ }^{1}$, Izabel Almeida Alves ${ }^{2}$

${ }^{1}$ Universidade Regional Integrada do Alto Uruguai e das Missóes (URI), Santo Ângelo, RS, Brasil; ${ }^{2}$ Universidade Federal da Bahia, Salvador, BA, Brasil.

\section{RESUMO}

Objetivo: Determinar os microrganismos contaminantes do ambiente hospitalar e avaliar o perfil de resistência a antimicrobianos dos isolados oriundos da unidade de terapia intensiva adulta de um hospital localizado na região noroeste do estado do Rio Grande do Sul. Métodos: Foram analisadas 216 amostras, coletadas das diferentes superfícies da unidade de terapia intensiva Adulta, em dois dias alternados, antes e depois da limpeza terminal. Os isolados foram identificados através de técnicas microbiológicas convencionais. Os testes de susceptibilidade foram realizados baseados no protocolo M100-S23 do CLSI, 2013. Resultados: Das 216 amostras, 115 (53,2 \%) apresentaram crescimento microbiológico. Os microrganismos que apresentaram maior prevalência foram Staphylococcus coagulase negativo $(43,4 \%)$, seguido por Staphylococcus aureus (20,5\%), Bacilos gram positivos (14,7\%), Acinetobacter iwofii (4,9\%), Pseudomonas aeruginosa (4,9 \%), Klebsiella pneumoniae (4,1\%), Acinetobacter baumanii (2,5 $\%)$, Acinetobacter calcoaceticus (1,7\%), Candida albicans, Escherichia coli e Citrobacter freundii $(0,82 \%)$. Dentre os isolados cocos gram positivos $27,4 \%$ apresentaram resistência a penicilina e 18,0 \% a clindamicina, já entre as enterobacterias 25,0 \% demonstraram resistência a amoxicilina e 14,3 \% a ceftriaxona. Conclusáo: Constatou-se que as maiores prevalências foram dos gêneros SCN, seguido por $S$. aureus, bem como, observou-se crescimento de microrganismos de importante patogenecidade. O perfil de sensibilidade demonstrou que as bactérias isoladas apresentam resistência a muito dos antibióticos utilizados. Diante dos fatos, a adoção de boas práticas de desinfecção e higiene são uma possibilidade para a redução da carga microbiana no ambiente hospitalar.

Descritores: Infecção Hospitalar; Bactérias; Unidade de Terapia Intensiva; Hospital. 


\section{ABSTRACT}

Objective: To determine the contaminating microrganisms of the hospital environment and to evaluate the antimicrobial resistance profile of isolates from the adult intensive care unit of a hospital. Methods: A total of 216 samples were collected from different surfaces of the adult ICU on two alternate days before and after terminal cleaning. Isolates were identified by conventional microbiological techniques. Susceptibility tests were performed based on CLSI protocol M100-S23, 2013. Results: Of the 216 samples, 115 (53.2\%) showed microbiological growth. The most prevalent microrganisms were coagulase-negative Staphylococcus (43.4\%), followed by Staphylococcus aureus (20.5\%), grampositive bacilli (14.7\%), Acinetobacter iwofi (4.9\%), Pseudomonas aeruginosa (4.9\%), Klebsiella pneumoniae (4.1\%), Acinetobacter baumanii (2.5\%), Acinetobacter calcoaceticus (1.7\%), Candida albicans, Escherichia coli and Citrobacter freundii (0.82\%). Among the gram-positive cocci isolates $27.4 \%$ showed resistance to penicillin and $18.0 \%$ to clindamycin, while among the enterobacteria $25.0 \%$ showed resistance to amoxicillin and $14.3 \%$ to ceftriaxone. Conclusion: In view of the observation of the spread of pathogens, particularly resistant ones, in a hospital environment, it is necessary to know more about environmental contaminants, in order to issue effective measures for the control of sources, dissemination pathways and availability of resources that enable disinfection. of these places.

Descriptors: Hospital Infection; Bacteria; Intensive care unit; Hospital.

\section{INTRODUÇÃO}

A Infecção Hospitalar (IH) é um a complicação muito recorrente e que afeta milhares de pessoas em todo o mundo. Devido a isso, as consequências ocasionadas tratam-se tanto de problemas éticos, jurídicos e sociais, quanto de custos relacionados ao prolongamento do tratamento e ao tempo de internaçáo, bem como, ao tempo de exposição do paciente a infecçóes e patógenos, o que em casos mais graves, pode levar ao aumento no número de óbitos ${ }^{1}$.

Segundo a Organização Mundial da Saúde (OMS), em países desenvolvidos há um índice de pelo menos 5\% dos pacientes internados em hospitais adquirirem algum tipo de infecção ${ }^{2}$. Vários são os fatores que induzem na proliferação dos patógenos na $\mathrm{IH}$, alguns destes são a sua origem, via de transmissão, o tipo do agente infeccioso e a susceptibilidade do ambiente para com o desenvolvimento do patógeno ${ }^{3}$.

Grande parte das IH são oriundas de forma endógena, ou seja, dos microrganismos presentes na microbiota do paciente, podendo ocorrer devido à progressão da doença ou ainda, devido aos procedimentos realizados para a descoberta de seu diagnóstico e a terapêutica pela qual o paciente foi submetido. Os demais causadores de IH encontramse na forma exógena, ou seja, os seus agentes procedentes originaram-se da água, do ar ou dos materiais hospitalares ${ }^{4}$.

Outra explicação para essas infecçóes é o uso inadequado dos medicamentos antimicrobianos, uma vez que o seu mal-uso torna-se uma ameaça diante da capacidade 
microbiana de desenvolvimento multirresistente ${ }^{3}$. O uso exacerbado de antimicrobianos, a reuniáo de diversas pessoas com a saúde vulnerável e os incontáveis procedimentos invasivos de fato, elevam o índice de presença de agentes infecciosos multirresistentes. As elevadas taxas de problemas relacionados à saúde é uma complicação a nível mundial, consequência do aumento da resistência destes microrganismos 5 .

O impacto das IH deve ser levado em consideração devido aos fatores que as mesmas desencadeiam, por isso, é fundamental a constituição da Comissão de Controle de Infecção Hospitalar (CCIH) presente nos hospitais, conforme segue na Portaria no 2.616/98, a fim de diminuir o índice de IH para aumentar a segurança e efetividade no tratamento do paciente ${ }^{6}$.

Segundo um estudo realizado por Lisboa e colaboradores (2007) foram coletados dados de 16 unidades de terapia intensiva (UTI) do estado do Rio Grande do Sul, em que para 174 pacientes pesquisados, 122 pacientes (71\%) estavam infectados, e 51 (29\%) adquiriram uma infecção na UTI. Destas, pneumonia foi a mais frequente $(58,2 \%)$, seguido pela infecção do trato respiratório inferior $(22,9 \%)$ e pela infecção do trato urinário (18\%). Os agentes causadores de infecçóes mais relatados foram Staphylococcus aureus (42\%) - 64\% resistentes a oxacilina - e Pseudomonas aeruginosa (31\%). Além disso, nesse estudo foram identificados os principais fatores de risco para o desenvolvimento de infecçóes adquiridas nas UTI’s: uso de cateter urinário, acesso vascular central, intubação traqueal por tempo prolongado ( $>4$ dias), doença crônica, trauma e internação prolongada na UTI (> 30 dias) $)^{7}$.

O objetivo do presente estudo foi determinar os microrganismos contaminantes do ambiente hospitalar e avaliar o perfil de resistência a antimicrobianos dos isolados oriundos da UTI adulta de um hospital da região noroeste do Rio Grande do Sul.

\section{MATERIAIS E MÉTODOS}

Trata-se de um estudo transversal, observacional e prospectivo, realizado em um hospital da região noroeste do Rio Grande do Sul, o qual dispóe do total de 180 leitos, sendo 9 leitos dispostos na UTI adulta, ambiente que conta com aproximadamente 100 $\mathrm{m}^{2}$. As coletas foram realizadas no mês de abril de 2013, por meio de amostras coletadas com swabs estéreis em diferentes superfícies das UTI adulta.

Foram coletadas 216 amostras, sendo os locais definidos conforme o protocolo "Controle da disseminação de Acinetobater sp resistente a carbapenêmicos no município de Porto Alegre" e adaptados conforme realidade da unidade em questão ${ }^{8}$. As amostras foram coletadas em dois dias alternados, no primeiro dia as amostras foram coletadas previamente a limpeza terminal do setor, e, no segundo dia foram coletadas amostras das superfícies posteriormente a limpeza. Para a busca ativa dos microrganismos 
contaminante do ambiente da UTI adulta, foram coletadas amostras dos seguintes locais da unidade: ventiladores mecânicos, válvulas de oxigênio, caixa de materiais individuais, armários individuais, bombas de infusão, monitores cardíacos, manivelas de elevação dos pacientes, grades das macas, colchóes, pias, dispensadores de sabonete líquido, bancadas de diluição e da enfermagem, teclados, vincos do rodapé, aparelho de hemodiálise e Raio-X. Perfazendo um total de 216 amostras coletadas. No primeiro dia de coleta foram coletadas 117 amostras, no segundo dia foram coletadas 99 amostras. O objetivo do estudo não foi avaliar a eficácia da limpeza terminal, sendo assim as amostras não foram analisadas separadamente.

As amostras coletadas com auxílio de swab estéril foram armazenadas em meio de transporte Stuart (Cralplast ${ }^{\circ}$. As amostras foram encaminhadas ao laboratório de Microbiologia da Universidade Regional Integrada do Alto Uruguai e das Missóes URI Campus de Santo Ângelo para isolamento e identificação dos microrganismos. Inicialmente as amostras foram semeadas em ágar sangue 5\% (Oxoid ${ }^{\circ}$ ) e ágar McConkey $\left(\mathrm{Oxoid}^{\circ}\right)$ e incubadas a $37^{\circ} \mathrm{C}$ por 24 horas.

Foram utilizados métodos microbiológicos convencionais para o isolamento e identificação dos microrganismos. Após a incubação, os bacilos gram-negativos fermentadores foram identificados através de provas bioquímicas (Mio, Lia, TSI, Citrato e Urease), os bacilos gram-negativos não-fermentadores utilizando o Kit comercial específico NFII (Probac do Brasil ${ }^{\circ}$ ) e os cocos gram-positivos através da realização dos testes da catalase, Staphy test (Probac do Brasil ${ }^{\circ}$ ) e coagulase em tubo.

Os testes de sensibilidade aos antimicrobianos foram realizados segundo o protocolo M100-S23 do Clinical and Laboratory Standards Institute através da técnica de Disco-difusão (Kirby-Bauer) em ágar Mueller-Hinton (Oxoid ${ }^{\circ}{ }^{9}$. Foram utilizados os seguintes discos de antibacterianos: Ampicilina (AMP), Cefalotina (CFL), Cefoxitina (CFO), Amicacina (AMC), Sulfametazol+Trimetroprima (SMX-TMP), Penicilina (PEN), Eritomicina (ERI), Clindamicina (CLI), Ciprofloxacino (CIP), Ceftazidima (CAZ), Oxacilina (OXA), Imipenem (IPM), Cefepima (CPM), Ampicilina+Sulbactam (SAM), Meropenem (MPM), Cefepime (CPM), Levofloxacino (LEV), Ceftriaxona (CRO), Gentamicina (GEN), Cefaclor (CFO). A escolha dos antibacterianos foi baseada na padronização de medicamentos da farmácia do hospital em estudo. Os halos foram medidos e interpretados de acordo com as normas do CLSI, $2013^{9}$.

\section{RESULTADOS}

Todos os locais e equipamentos avaliados apresentaram crescimento microbiológico, em ambos os dias de coleta, conforme a Tabela 1. Com exceçáo do segundo dia, aonde não houve crescimento de microrganismos na bancada de diluição 
e equipamento de hemodiálise. Das 216 amostras coletadas, 115 (53,2\%) apresentaram crescimento microbiano, sendo identificados 122 microrganismos. Na coleta realizada antes da limpeza terminal do setor, houve crescimento microbiano em 68 (58,1\%) amostras. Após a limpeza terminal, houve crescimento em 47 (47,5\%).

A prevalência dos microrganismos isolados neste estudo, em ambos os dias, foram: Staphylococcus coagulase negativo (SCN) 43,4\% (53/122), Sthapylococcus aureus 20,5\% (25/122), Bacillus spp. 14,8\% (18/122), Acinetobacter Iwoffii 5,7\% (7/122), Pseudomonas aeruginosa 4,9\% (6/122), Klebsiella pneumoniae 4,1\% (5/122), Acinetobacter baumanii 2,5\% (3/122), Acinetocacter calcoaceticus 1,6\% (2/122) e Citrobacter freundii, Candida albicans e Escherichia coli com 0,8\% (1/122) cada microrganismo (Tabela 2). Do total das amostras que apresentaram crescimento microbiano, em 10 foram observados o isolamento de mais de um microrganismo.

Tabela 1. Locais de coleta, número de amostras coletadas e número de amostra colonizadas.

\begin{tabular}{|c|c|c|}
\hline Locais de coleta & Número de amostras & $\begin{array}{c}\text { Número de amostras } \\
\text { colonizadas }\end{array}$ \\
\hline Ventiladores Mecânicos & 19 & 10 \\
\hline Válvulas de Oxigênio & 20 & 5 \\
\hline Caixas de materiais individuais & 20 & 13 \\
\hline Armários individuais & 20 & 15 \\
\hline Bombas de infusão & 19 & 7 \\
\hline Monitores cardíacos & 20 & 9 \\
\hline Manivelas de elevação & 11 & 4 \\
\hline Grades das macas & 11 & 6 \\
\hline Colchóes & 20 & 5 \\
\hline Pias & 10 & 8 \\
\hline Torneiras & 10 & 8 \\
\hline Dispensadores & 10 & 5 \\
\hline Bancadas de Enfermagem & 3 & 3 \\
\hline Bancadas de Diluição & 3 & 2 \\
\hline Teclados & 6 & 5 \\
\hline Hemodiálise & 6 & 2 \\
\hline Vincos do rodapé & 6 & 6 \\
\hline Raio X & 2 & 2 \\
\hline TOTAL & 216 & 115 \\
\hline
\end{tabular}

Quando analisado o perfil de resistência dos cocos gram-positivos (SCN e $S$. aureus) $60,3 \%$ mostraram-se resistentes a penicilina; $37,7 \%$ a clindamicina; $37,2 \%$ a 
eritromicina; 32,1\% a Sulfametozaxol+Trimetroprim; 26,9\% a Cefoxitina e Oxacilina, e 24,3\% a Ciprofloxacino (Tabela 3).

Tabela 2. Frequência e prevalência de microrganismos isolados dos materiais e locais da UTI.

\begin{tabular}{lcc}
\hline Microrganismos & N & \% \\
\hline Staphylococcus coagulase negativo & 53 & $43,4 \%$ \\
\hline Sthaphylococcus aureus & 25 & $20,5 \%$ \\
\hline Bacillus spp. & 18 & $14,8 \%$ \\
\hline Acinetobacter Iwoffi & 7 & $5,7 \%$ \\
\hline Pseudomonas aeruginosa & 6 & $4,9 \%$ \\
\hline Klebsiella pneumoniae & 5 & $4,1 \%$ \\
\hline Acinetobacter baumanii & 3 & $2,5 \%$ \\
\hline Acinetobacter calcoaceticus & 2 & $1,6 \%$ \\
\hline Candida albicans & 1 & $0,8 \%$ \\
\hline Citrobacter freundii & 1 & $0,8 \%$ \\
\hline Escherichia coli & 1 & $0,8 \%$ \\
\hline TOTAL & $\mathbf{1 2 2}$ & $\mathbf{1 0 0 , 0 0 \%}$ \\
\hline
\end{tabular}

Tabela 3. Perfil de resistência dos cocos gram-positivos (S. aureus e $\left.\mathrm{SCN}^{*}\right)$ isolados dos materiais e locais da UTI $(\mathrm{n}=78)$.

\begin{tabular}{lcc}
\hline \multicolumn{1}{c}{ Antimicrobianos } & Número de isolados resistentes & \% de isolados resistentes \\
\hline Penicilina & 47 & $60,25 \%$ \\
\hline Clindamicina & 31 & $37,74 \%$ \\
\hline Eritromicina & 29 & $37,17 \%$ \\
\hline Sulfazotrim & 25 & $32,05 \%$ \\
\hline Cefoxitina & 21 & $26,92 \%$ \\
\hline Oxaciclina & 21 & $26,92 \%$ \\
\hline Ciprofloxacino & 19 & $24,35 \%$ \\
\hline
\end{tabular}

*SCN $=$ Staphylococcus coagulase negativo

Analisando o perfil de sensibilidade das enterobactérias observou-se resistência prevalente a amoxicilina (100\%), a ceftriaxona (57,1\%) e a ampicilina/sulbactam $(57,1 \%)$. As bactérias manifestaram sensibilidade total aos antimicrobianos gentamicina e levofloxacino (Tabela 4). As enterobactérias apresentaram resistência inferior aos demais antimicrobianos.

Os bacilos gram-negativos não-fermentadores Acinetobacter ssp. e P. aeruginosa apresentaram resistência a gentamicina $(44,4 \%)$, a amicacina $(44,4 \%)$ e a cefepime $(33,3 \%)$. Os antimicrobianos levofloxacino, ceftriaxona, ampicilina/sulbactram e meropenem apresentam total sensibilidade aos bacilos gram-negativos não-fermentadores (Tabela 5). 
Tabela 4. Perfil de resistência das enterobactérias (K. pneumoniae, E. coli e C. freundii) isolados dos materiais e locais da UTI $(\mathrm{n}=7)$.

\begin{tabular}{lcc}
\hline \multicolumn{1}{c}{ Antimicrobiano } & Número de isolados resistentes & \% de isolados resistentes \\
\hline Amoxicilina & 7 & $100,0 \%$ \\
\hline Ceftriaxona & 4 & $57,1 \%$ \\
\hline Ampicilina/Sulbactam & 4 & $57,1 \%$ \\
\hline Meropenem & 3 & $42,8 \%$ \\
\hline Ceftazidima & 3 & $42,8 \%$ \\
\hline Ciprofloxacino & 2 & $28,6 \%$ \\
\hline Amicacina & 1 & $14,3 \%$ \\
\hline & 1 & $14,3 \%$ \\
\hline Piperaciclina/Tazobactam & 1 & $14,3 \%$ \\
\hline Cefepime & 1 & $14,3 \%$ \\
\hline Cefaclor & 1 & $14,3 \%$ \\
\hline Cefazolina & 0 & $0,0 \%$ \\
\hline Gentamicina & 0 & $0,0 \%$ \\
\hline Levofloxacino & &
\end{tabular}

Tabela 5. Perfil de resistência dos bacilos gram-negativos não-fermentadores (Acinetobacter ssp. $e$ P. aeruginosa) isolados dos materiais e locais da UTI $(\mathrm{n}=18)$.
Antimicrobiano
Número de isolados resistentes
$\%$ de isolados resistentes

\begin{tabular}{lll}
\hline Gentamicina & 8 & $44,4 \%$ \\
\hline Amicacina & 8 & $44,4 \%$ \\
\hline Cefepime & 6 & $33,3 \%$ \\
\hline
\end{tabular}

\begin{tabular}{lll} 
Piperaciclina/Tazobactam & 2 & $10,5 \%$ \\
\hline Ciprofloxacino & 1 & $5,6 \%$ \\
\hline Ceftazidima & 1 & $5,6 \%$ \\
\hline Levofloxacino & 0 & $0,0 \%$ \\
\hline Ceftriaxona & 0 & $0,0 \%$ \\
\hline Ampicilina/Sulbactam & 0 & $0,0 \%$ \\
\hline Meropenem & 0 & $0,0 \%$ \\
\hline
\end{tabular}




\section{DISCUSSÃO}

A IH é uma condição infecciosa que pacientes hospitalizados estáo expostos, devido ao seu tempo de internação e aos procedimentos aos quais o mesmo é submetido. $\mathrm{O}$ prolongamento do tempo de internação é um agravante a esses pacientes, principalmente aos internados nas UTI`s, visto que, estimativas afirmam que ao menos $5 \%$ dos pacientes internados em hospitais adquirem uma infecção ${ }^{1,10}$.

Os pacientes internados nas UTIs, em sua maioria, são submetidos a procedimento invasivos que acarretam na irrupção dos tecidos subcutâneos, da derme e da epiderme, promovendo a exposiçáa dos órgãos internos do indivíduo a potenciais patógenos, sejam esses devido à migração dos microrganismos através de uma contaminação cruzada contaminaçáo exógena - ou através da microbiota do próprio paciente - contaminação endógena. Além disso, fatores de risco como a imunossupressáo dos mesmos deve ser levada em consideração, pois a facilidade de proliferaçấo de microrganismos patológicos é alta ${ }^{11}$.

Diante disto, a limpeza e sanitização dos ambientes hospitalares, principalmente das UTIs são imprescindíveis. Produtos com ação antimicrobiana auxiliam na eliminação dos microrganismos presentes no local, da mesma forma que medidas simples como a higienização das mãos evitam a proliferação dos mesmos e diminuem as taxas de $\mathrm{IH}^{12}$.

As UTIs são áreas onde frequentemente são encontradas contaminaçôes em seu ambiente e nas superfícies, devido ao grande número de profissionais da saúde atuando 24 horas por dia no cuidado dos pacientes em estado grave, aumentando consequentemente, o contato das mãos de tais profissionais com os equipamentos e superfícies, ampliando o risco de contaminaçáa ${ }^{13}$. Neste hospital, a limpeza terminal da UTI é realizada semanalmente, na saída do paciente ou sempre que necessário. Os pisos e as paredes são limpos com hipoclorito de sódio $1 \%$, as superfícies com álccol $70 \%$.

Estudos demonstram a prevalência de microrganismos relevantes clínica e epidemiologicamente em superfícies secas por dias, ou até, mais de um ano, como ocorre no caso da E. coli com um tempo de sobrevivência de $2 \mathrm{~h}$ até 16 meses, o mesmo ocorre com a Klebsiella sp., podendo sobreviver entre $2 \mathrm{~h}$ até 30 meses $^{14}$. Dessa forma, materiais utilizados diariamente pelos profissionais da saúde e pelos pacientes são afetados, como telefones, bancadas, teclado de computador, colchôes, eletrocardiograma e demais equipamentos em geral, além dos materiais hospitalares como cateter, válvulas de oxigênio, bomba de infusão, entre outros instrumentos os quais podem ser contaminados ${ }^{13}$.

O S. aureus e o SCN foram os microrganismos mais isolados, para tanto, autores como Silva, 2019 (14) destacam que o corpo humano e a sua microbiota natural possuem mais microrganismos do que células ${ }^{14}$. Em contrapartida, Mendes e Brasileiro, 2017 (13) enfatizam a importância dos cuidados de assepsia para a reduçáo da contaminação por 
meio da higienização das mãos antes e, após contato com os pacientes. A falta de assepsia por parte dos trabalhadores que atuam em hospitais pode ser uma forma de condução de infecçóes do ambiente hospitalar para a comunidade e vice-versa ${ }^{13}$.

Estudos feitos por Amador, Basso e Vieira (10) apresentam crescimento positivo para S. aureus em 50\% das amostras coletadas em 25 superfícies da UTI de um hospital na cidade de Umuarama-PR, sendo essas, amostras de leitos, prontuários, portas de entrada, teclado do computador, banheiro dos funcionários, bancada direita e esquerda ${ }^{10}$.

Em nossos estudos, das 216 amostras coletadas em superfícies, 115 (53,24\%) apresentaram crescimento microbiano, sendo identificados 122 microrganismos. Destes, 20,5\% (25/122) apresentaram crescimento positivo para $S$. aureus, demonstrando número inferior de superfícies colonizadas, se comparado aos resultados do estudo feito por Amador, Basso e Vieira (10).

Dentre os microrganismos mais prevalentes nas IHs encontra-se as bactérias do gênero Acinetobacter spp., esta que é classificado entre as espécies Acinetobacter calcoacetius, Acinetobacter baumannii, Acineteobacter pitti e Acineteobacter nosocomialis. Um dos motivos da sua alta prevalência é a sua resistência a antibióticos, resultando na sua predominância em diferentes ambientes e locais ${ }^{15}$.

Estudos feitos nos Estados Unidos mostram que a espécie mais prevalente de IHs em UTIs são do gênero A. baumannii. Estudos abordados por Araújo (15) relatam que 10 a 43\% dos pacientes internados nas UTIs são infectados por essa bactéria, causando uma mortalidade de 7,8 a $23 \%$ desses pacientes. Acredita-se que um dos motivos para a sua elevada prevalência se dá pela permanência da $A$. baumannii no organismo em cerca de 3 dias a 5 meses $^{15}$. Nosso estudo teve prevalência de $2,5 \%$ de $A$. baumannii, as cepas isoladas apresentaram resistência a gentamicina, ceftriaxona e amicacina. Estudos feitos por Lima, 2019 (16) demonstram que todas as cepas isoladas de A. baumannii coletadas em superfícies e das máos de profissionais da UTI de um hospital brasileiro demostraram característica pan-resistente aos antibióticos testados ${ }^{16}$.

Estudos feitos por Mota, Oliveira e Souto (17) citam que segundo a OMS, a $P$. aeruginosa é classificada como prioridade crítica de resistência, necessitando urgentemente do desenvolvimento de novos antibióticos. Nesse estudo foram feitas coletas dos pacientes da UTI do Hospital Santa Casa de Misericórdia de Goiânia, que demonstraram uma prevalência da $P$. aeruginosa em $11 \%$ das amostras. Entretanto, as amostras contendo a $P$. aeruginosa apresentaram resistência de 33,3\% à meropenem; de 22,2\% à ciprofloxacina e de $18,5 \%$ à gentamicina, cefepima e amicacina. A $P$. aeruginosa apresentou resistência intrínseca ao antimicrobiano ampicilina/sulbactam, porém, apresentou resistência nula a ceftzidima ${ }^{17}$.

Contudo, o nosso estudo demonstrou a presença da $P$. aeruginosa em 4,9\% das amostras coletadas de materiais hospitalares. As amostras contendo a $P$ a aeruginosa 
apresentaram-se resistentes a ciprofloxacina $(5,5 \%)$, a gentamicina $(44,4 \%)$, a amicacina $(5,5 \%)$, a cefepima $(33,3 \%)$ e a ceftzidima (5,5\%). Os antimicrobianos ampicilina/ sulbactam, meropenem, ceftriaxona e levofloxacino apresentaram sensibilidade total contra a $P$. aeruginosa.

No atual estudo a $K$. pneumoniae foi presente em 4,1\% das amostras coletadas, apresentando total resistência a amoxicilina, 57,1\% resistente à ceftriaxona e ampicilina, seguido de 42,8\% resistente à meropenem e ceftazidima. Os antimicrobianos gentamicina e levofloxacino apresentaram sensibilidade total à K. pneumoniae.

Estudos da literatura demonstram que entre os andares eblocos onde foram coletadas amostras, houve a prevalência do crescimento de $K$. pneumoniae na UTI, com $28,6 \%$ do total de 133 isolados. Estes resultados evidenciam a ocorrência do desenvolvimento de fatores de resistência bacteriana da $K$. pneumoniae e, consequentemente, na insuficiência de ação dos antimicrobianos ${ }^{18}$. Devido ao nosso estudo e aos estudos citados da literatura serem feitos em diferentes regióes, a variação de percentual pode ocorrer, entretanto, em todos os estudos se observa a prevalência da $K$. pneumoniae em isolados de UTI.

Dentre os microrganismos com menor prevalência, no presente estudo, estão a $E$. coli, C. albicans e C. freundii, ambos com prevalência de $0,8 \%$.

Mota, Oliveira e Souto, 2018, realizaram um estudo demonstrando a presença de $0,8 \%$ da prevalência de $C$. freundii nas amostras coletadas de UTI na cidade de Goiânia ${ }^{17}$.

As infecções por Candida em pacientes hospitalizados estão em crescimento nas últimas três décadas. Pacientes em longa hospitalização, internados em UTIs, com cateteres urinários, diagnosticados com diabetes mellitus ou pacientes imunocomprometidos são alguns dos fatores de risco para a incidência da Candida nas infecçóes do trato urinário (ITU). A espécie mais frequente que causa ITU é a $C$. albicans, esta que, apesar da sua baixa taxa de mortalidade, apresenta alta morbidade, principalmente se tratando de pacientes em UTIs ${ }^{19}$.

Em relação a $E$. coli, conforme foi possível observar nas tabelas, nossos estudos resultaram em uma baixa colonização, com $0,8 \%$, à vista disso, os trabalhos descritos na literatura científica diferem dos observados no atual estudo. A literatura demonstra que as amostras coletadas em pacientes internados na UTI e no pronto socorro clínico de um hospital em Goiânia, apresentam 39,6\% de prevalência de E. coli $i^{17}$. Este resultado mostra a importância deste tipo de estudo acerca do conhecimento da colonização de bactérias em ambientes hospitalares. Observou-se que os hospitais onde foram realizados os estudos encontrados na literatura, possuem uma elevada colonização de bactérias causadoras de infecções de vias aéreas superiores, especialmente, levando em consideração que muitos pacientes internados em UTIs estão entubados e sobre ventilação mecânica, circunstâncias as quais são fatores de risco para este tipo de infecção ${ }^{17,20,21}$. 


\section{CONCLUSÃO}

Através do presenteestudo foi possível determinarosmicrorganismoscontaminantes do ambiente da UTI hospitalar e avaliar o perfil de resistência a antimicrobianos. Constatouse que metade das amostras coletadas apresentaram crescimento microbiológico, cujo as maiores prevalências foram dos gêneros $\mathrm{SCN}$, seguido por $S$. aureus. Além disso, observou-se crescimento de microrganismos de importante patogenicidade para pacientes internados em UTIs, tais como K. pneumoniae, P. aeruginosa e Acinetobacter spp., entre outros. O perfil de sensibilidade demonstrou que neste ambiente hospitalar as bactérias isoladas apresentam resistência a muito dos antibióticos utilizados para tratamento das infecçóes hospitalares e ambulatoriais.

Portanto, para a redução da carga microbiana nas superfícies, deve-se adquirir práticas assíduas para a limpeza e descontaminação de equipamentos e profissionais. Diante disto, vale investimento em programas educativos para os profissionais de saúde tendo como alvo os processos de limpeza hospitalar do ambiente e demais cuidados. Diante dos fatos, a adoção de boas práticas de desinfecção e higiene são uma possibilidade de reduzir a transferência de patógenos entre profissional, paciente e ambiente.

\section{REFERÊNCIAS}

1. GIROTI, A. L. B. et al. Programas de Controle de Infecção Hospitalar: Avaliação de indicadores de estrutura e processo. Revista da Escola de Enfermagem da USP. Rev Esc Enferm USP · 2018; 52. Acesso em: 17 Set 2019. Disponível em: DOI: https://doi. org/10.1590/s1980-220x2017039903364.

2. MENEGUETI, M. G. et al. Avaliação dos Programas de Controle de Infecção Hospitalar em serviços de saúde. Revista Latino Americana de Enfermagem v. 23, n. 1, p. 98-110. 2015. Acesso em: 17 Set. 2019. Disponível em: DOI: 10.1590/01041169.0113 .2530

3. NASCIMENTO, D. O.; SANTOS, L. A. Infecção relacionada a saúde: percepção dos profissionais de saúde sobre seu controle. Revista Interdisciplinar. v. 9, n. 2, p. 127-135, abr. mai. jun. 2016. Acesso em: 17 Set 2019. Disponível em: https:// revistainterdisciplinar.uninovafapi.edu.br/index.php/revinter/article/view/929

4. SILVA, P. L. N. et al. Prevenção de Infecções Hospitalares em Pacientes Internados em Unidade de Terapia Intensiva. Revista da Universidade Vale do Rio Verde, Três Coraçóes, v. 13, n. 2, p. 649-658, 2015.

5. FERRAZ, C. C. B. et al. Fatores Associados a Infecçóes Hospitalares Causadas por Microrganismos Multirresistentes num Hospital de Ensino. Perspectivas Experimentais e Clínicas, Inovações Biomédicas e Educação em Saúde - PECIBES, v. 2, p. 52- 
57, 2016. Acesso em: 05 Out 2019. Disponível em: https://pdfs.semanticscholar. org/8491/ea1df54a72dfa6e201a3afdf669e13f5cc02.pdf

6. BRASIL, Ministério da saúde. Portaria GM/MS no 2.616, de 12 de maio de 1998. Dispóe sobre diretrizes e normas para a prevenção e o controle das infecçóes hospitalares [Internet]. Brasília; 1998. Acesso em: 05 Set 2019. Disponível em: http:// bvsms.saude.gov.br/bvs/saudelegis/gm/1998/prt2616_12_05_1998

7. VERLI, M. V.; DE OLIVEIRA, L. C.. Uma visão ampla das infecçóes hospitalares. Revista Panorâmica online, v. 27, n. 2, 2019. Acesso em: 15 Out 2019. Disponível em: https://www.researchgate.net/publication/335774768_UMA_VISAO_ AMPLA_DAS_INFECCOES_HOSPITALARES

8. RIO GRANDE DO SUL. Secretaria de Saúde. Manual de Orientação para Controle da Disseminação de Acinetobacter sp Resistente a Carbapenêmicos no Município de Porto Alegre, Porto Alegre, maio, 2007, p.6.

9. CLSI. Performance standards for antimicrobial susceptibility testing. m100-s23th. Clinical and Laboratory Standards Institute, Wayne, PA., 2013

10. AMADOR, J. F. S.; BASSO, L. C.; VIEIRA, S. L. V. Prevalência de Staphylococcus aureus em superfícies de unidade de terapia intensiva. Arquivos do Museu Dinâmico Interdisciplinar, v. 22, n. 2, p. 1-10, 2018. Acesso em: 15 Out 2019. Disponível em: DOI: https://doi.org/10.4025/arqmudi.v22i2.42861

11. ARAÚJO, A. R. A. Análise dos fatores de risco do diagnóstico de enfermagem risco de infecção em pacientes com câncer hospitalizados. 2016. Dissertação de Mestrado. Brasil. Acesso em: 15 Out. 2019. Disponível em: https://repositorio.ufrn.br/jspui/ handle/123456789/22430

12. GILDO, M. G. P. et al. Avaliação da eficácia antimicrobiana de desinfetantes utilizados na rotina de limpeza hospitalar. Revista Expressão Católica Saúde, v. 2, n. 2, p. 34-39, 2018. Acesso em: 20 Dez 2019 Disponível em: DOI: 10.25191/recs. v2i2.2200

13. MENDES, J. R.; BRASILEIRO, M. S. E. Proposta de protocolo para descontaminação de equipamentos em unidade de terapia intensiva. Revista de Enfermagem do Centro-Oeste Mineiro, v. 7, 2017. Acesso em: 19 Dez 2019. Disponível em: DOI: https://doi.org/10.19175/recom.v7i0.2346

14. SILVA, L. N. et al. Avaliação da contaminaçáo de pranchetas em uso em unidades de terapia intensiva. Dissertação de Mestrado. Ciências da Saúde, Universidade Federal de Goiás, Goiânia 2019. Acesso em: 19 Dez 2019. Disponível em: http://repositorio. bc.ufg.br/tede/handle/tede/10321 
15. ARAÚJO, A. A. Otimização aplicada ao processo de transmissão de Acinetobacter spp em unidades de terapia intensiva. Dissertação de mestrado, Universidade Estadual Paulista. 2018. Acesso em: 19 Dez 2019. Disponível em: http://hdl.handle. net/11449/181504

16. LIMA, L. K. O. L. et al. Avaliação da contaminação por Acinetobacter spp. em uma unidade de terapia intensiva. Revista epidemiologia e controle de infecção, p. 241247, 2019. Acesso em 20 Dez 2019. Disponível em: DOI: http://dx.doi.org/10.17058/ reci.v9i3.12510

17. MOTA, F. S.; OLIVEIRA, H. A.; SOUTO, R. C. F. Perfil e prevalência de resistência aos antimicrobianos de bactérias Gram-negativas isoladas de pacientes de uma unidade de terapia intensiva. Revista Brasileira de Análises Clínicas, v. 50, n. 3, p. 270-7, 2018. Acesso em: 12 Fev 2020. Disponível em: DOI: 10.21877/24483877.201800740

18. GOMES, D. R. M.; COPETTI, C. Características Microbiologicas de Klebsiella pneumoniae Isoladas em Meio Ambiente Hospitalar: Uma Revisão. Revista Saúde Integrada, v. 11, n. 22, p. 71-84, 2018. Acesso em: 12 Fev 2020. Disponível em: http:// local.cnecsan.edu.br/revista/index.php/saude/article/view/582

19. RISHI S, JAIN B, RUCHI et.al. Prevalence of albicans and non-albicans candiduria in a tertiary care hospital of Jaipur, India. Gal Int J Health Sci Res. 2020; 5(1): $1-5$.

20. RODRIGUES, J. M. R. S. et al. Prevalência e perfil de sensibilidade dos agentes microbianos causadores de infecçóes de trato urinário. Revista Brasileira de Análises Clínicas v. 49, n. 4, p. 381-9 2018. Disponível em: DOI: 10.21877/24483877.201700580

21. BARBOSA, L. R.; MOTA, É. C.; OLIVEIRA, A. C. Urinary tract infection associated with bladder catheter in intensive care unit. Revista de Epidemiologia de Controle de Infecção, v. 9, n. 2, p. 103-108, 2019. Acesso em: 12 Fev. 2020. Disponível em: DOI: http://dx.doi.org/10.17058/reci.v9i1.11579

Autor Correspondente: Izabel Almeida Alves E-mail: izabelalmeidaalves@gmail.com

Recebido em: 2020-04-08 Aprovado: 2020-06-16 